\title{
Galaxies in most dense environments at redshift 1.4
}

\author{
V. Strazzullo \\ ${ }^{1}$ Irfu/SAp, CEA-Saclay, Orme des Merisiers, 91191 Gif sur Yvette, France \\ email: veronica.strazzullo@cea.fr
}

\begin{abstract}
At a cosmic time when galaxy clusters start showing evidence of a still active galaxy population, the X-ray luminous, massive cluster XMMU J2235-2557 at $z=1.39$, already hosts massive, quiescent, early-type galaxies on a tight red sequence dominating the cluster core. XMMU J2235-2557 is among the most massive of the very distant clusters, which may explain the evolved status of the system itself, and of its host galaxy populations. It remains a unique laboratory to observe environment-biased galaxy evolution already 9 billion years ago.
\end{abstract}

Keywords. Galaxies: clusters, Galaxies: evolution, Galaxies: high-redshift

The massive cluster XMMU J2235-2557 at $z=1.39$ already hosts evolved galaxy populations, with star formation effectively suppressed in massive galaxies, and confined outside of the cluster core. The luminosity/stellar mass functions suggest a stellar mass distribution similar to that of cluster galaxies at lower redshifts. A tight red sequence is already in place, its color and slope in agreement with passive evolution predictions with a formation redshift $z \sim 3$. The red sequence is mainly populated by massive galaxies with no evidence of star formation and early-type morphologies. The smaller sizes of these seemingly passively evolving objects compared to their $\mathrm{z}=0$ counterparts might leave room for later evolution, but its actual relevance for individual galaxies remains unclear due to uncertainties and biases in the masses, sizes, local mass-size relation, and in the comparison of samples at different cosmic epochs (see Strazzullo et al. 2010).
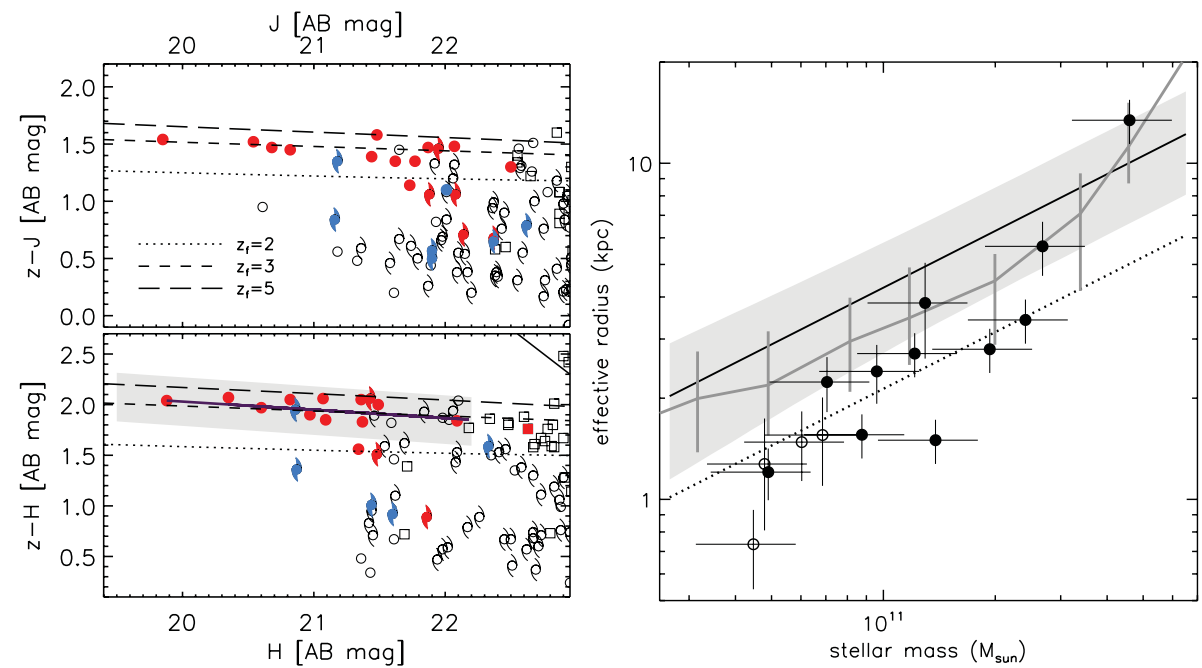

Figure 1. The red sequence (left), and stellar mass vs size relation for early-types (right), in the galaxy cluster XMMU J2235-2557 at z=1.39 (from Strazzullo et al. 2010). 